\title{
CONSTRUCTION OF VECTOR VALUED MODULAR FORMS FROM JACOBI FORMS
}

\author{
JAE-HYUN YANG
}

\begin{abstract}
We give a geometrical construction of the canonical automorphic factor for the Jacobi group and construct new vector valued modular forms from Jacobi forms by differentiating them with respect to toroidal variables and then evaluating at zero.
\end{abstract}

\section{INTRODUCTION}

For given two fixed positive integers $n$ and $m$, we let

$$
H_{n}:=\left\{Z \in \mathbb{C}^{(n, n)} \mid Z={ }^{t} Z, \quad \operatorname{Im} Z>0\right\}
$$

be the Siegel upper half plane of degree $n$ and let $\Gamma_{n}$ be the Siegel modular group of degree $n$. Let

$$
\mathcal{P}_{m, n}:=\mathbb{C}\left[W_{11}, \cdots, W_{m n}\right], \quad W=\left(W_{k l}\right) \in \mathbb{C}^{(m, n)}
$$

be the ring of polynomial functions on $\mathbb{C}^{(m, n)}$. Here $\mathbb{C}^{(n, n)}\left(\right.$ resp. $\left.\mathbb{C}^{(m, n)}\right)$ denotes the space of all complex $n \times n($ resp. $m \times n)$-matrices ( see notations below). For any homogeneous polynomial $P \in \mathcal{P}_{m, n}$, we define the differential operator $P\left(\partial_{W}\right)$ on $\mathbb{C}^{(m, n)}$ as follows:

$$
P\left(\partial_{W}\right):=P\left(\frac{\partial}{\partial W_{11}}, \cdots, \frac{\partial}{\partial W_{m n}}\right) .
$$

In this paper, the author proves that if $P$ is a homogeneous pluriharmonic polynomial in $\mathcal{P}_{m, n}$ and $f \in J_{\rho, \mathcal{M}}\left(\Gamma_{n}\right)$ (see Definition 3.1) is a Jacobi form of index $\mathcal{M}$ with respect to a rational representation $\rho$ of the general group $G L(n, \mathbb{C})$, then the following function

$$
\left.P\left(\partial_{W}\right) f(Z, W)\right|_{W=0}
$$

This work was in part supported by TGRC-KOSEF and Max-Planck-Institut für Mathematik. 1991 Mathematics Subject Classification. Primary 11F30, 11F55.

Typeset by $\mathcal{A M}_{\mathcal{M}} \mathcal{S}_{\mathrm{E}} \mathrm{X}$ 
yields a vector valued modular form with respect to a new rational representation of $G L(n, \mathbb{C})$. For a precise detail, we refer to Definition 5.1 and Main Theorem in section 5. In [M-N-N] (cf. pp 147-156), the authors proved the similiar result for theta functions. Our result is a generalization of their result because theta functions are special examples of Jacobi forms.

This paper is organized as follows. In section 2, we provide a geometrical construction of the canonical automorphic factor for the Jacobi group. In section 3, we review Jacobi forms and establish the notations. In section 4, we review pluriharmonic polynomials and obtain some properties to be used in the subsequent sections. In section 5 , we shall prove the main theorem. In section 6 , we obtain two identities by applying the main theorem to Jacobi forms.

Notations: We denote by $\mathbb{Z}, \mathbb{R}$ and $\mathbb{C}$ the ring of integers, the field of real numbers, and the field of complex numbers respectively. $\Gamma_{n}:=S p(n, \mathbb{Z})$ denotes the Siegel modular group of degree $n$. The symbol ":=" means that the expression on the right is the definition of that on the left. We denotes by $\mathbb{Z}^{+}$the set of all positive integers. $F^{(k, l)}$ denotes the set of all $k \times l$ matrices with entries in a commutative ring $F$. For a square matrix $A \in F^{(k, k)}$ of degree $k, \sigma(A)$ denotes the trace of $A$. For $A \in F^{(k, l)}$ and $B \in F^{(k, k)}$, we set $B[A]={ }^{t} A B A$. For any $M \in F^{(k, l)},{ }^{t} M$ denotes the transpose matrix of $M . E_{n}$ denotes the identity matrix of degree $n$.

\section{The Canonical Automorphic Factor for the Jacobi Group}

Let $m$ and $n$ be two fixed positive integers. It is well known that the automorphism group Aut $\left(H_{m+n}\right)$ of the Siegel upper half plane of degree $m+n$ is given by

$$
\text { Aut }\left(H_{m+n}\right)=S p(m+n, \mathbb{R}) /\left\{ \pm E_{m+n}\right\} .
$$

We observe that $H_{n}$ is a rational boundary of $H_{m+n}$ (cf. [N]). The normalizer $N\left(H_{n}\right):=\left\{\tilde{\sigma} \in \operatorname{Aut}\left(H_{m+n}\right): \tilde{\sigma}\left(H_{n}\right) \subset H_{n}\right\}$ of $H_{n}$ is given by

$$
N\left(H_{n}\right)=P\left(H_{n}\right) /\left\{ \pm E_{m+n}\right\}
$$

where

$$
\begin{aligned}
P\left(H_{n}\right): & =\left\{g \in S p(m+n, \mathbb{R}): g\left(H_{n}\right) \subset H_{n}\right\} \\
& =\{[\sigma, u,(\lambda, \mu, \kappa)] \in S p(m+n, \mathbb{R})\} .
\end{aligned}
$$

Here we put

$$
[\sigma, u,(\lambda, \mu, \kappa)]:=\left(\begin{array}{cccc}
A & 0 & B & A^{t} \mu-B^{t} \lambda \\
u \lambda & u & u \mu & u \kappa \\
C & 0 & D & C^{t} \mu-D^{t} \lambda \\
0 & 0 & 0 & { }^{t} u^{-1}
\end{array}\right)
$$


where $\sigma=\left(\begin{array}{ll}A & B \\ C & D\end{array}\right) \in S p(n, \mathbb{R}), u \in G L(m, \mathbb{R}), \lambda, \mu \in \mathbb{R}^{(m, n)}$ and $\kappa \in \mathbb{R}^{(m, m)}$. write

If $\left(\begin{array}{cc}Z & { }^{t} W \\ W & T\end{array}\right) \in H_{m+n}$ with $Z \in H_{n}, W \in \mathbb{R}^{(m, n)}$ and $T \in H_{m}$, we simply

$$
(Z, W, T):=\left(\begin{array}{cc}
Z & { }^{t} W \\
W & T
\end{array}\right)
$$

We denote the symplectic action of $N\left(H_{n}\right)$ on $(Z, W, T)$ by

$$
g \cdot(Z, W, T):=(\tilde{Z}, \tilde{W}, \tilde{T}), \quad g \in N\left(H_{n}\right) .
$$

It is easy to see that $(\tilde{Z}, \tilde{W}, \tilde{T})$ is of the form

$$
\begin{aligned}
\tilde{Z} & =\sigma_{g}(Z), \\
\tilde{W} & =a(g ; Z)(W)+b(g ; Z), \\
\tilde{T} & =m_{g}(T)+c(g ; Z, W),
\end{aligned}
$$

where $\sigma_{g} \in \operatorname{Aut}\left(H_{n}\right), m_{g} \in \operatorname{Aut}\left(\mathcal{P}_{m}\right)$,

$$
\begin{aligned}
& a(g ; \cdot): H_{n} \longrightarrow G L\left(\mathbb{C}^{(m, n)}\right) \text { holomorphic, } \\
& b(g ; \cdot): H_{n} \longrightarrow \mathbb{C}^{(m, n)} \quad \text { holomorphic, } \\
& c(g ; \cdot, \cdot): H_{n} \times \mathbb{C}^{(m, n)} \longrightarrow H_{m} \text { holomorphic. }
\end{aligned}
$$

Here $\mathcal{P}_{m}:=\left\{Y \in \mathbb{R}^{(m, m)} \mid Y={ }^{t} Y>0\right\}$ is an open convex cone in $\mathbb{R}^{\frac{m(m+1)}{2}}$ and we set

$$
\operatorname{Aut}\left(\mathcal{P}_{m}\right):=\left\{\xi \in G L\left(\mathbb{C}^{(m, m)}\right) \mid \xi\left(\mathcal{P}_{m}\right)=\mathcal{P}_{m}\right\} .
$$

REMARK 2.1. In [PS], Piateski-Sharpiro called the mapping $(Z, W, T) \longmapsto(\tilde{Z}, \tilde{W}, \tilde{T})$ a quasilinear transformation.

From now on, we set

$$
H_{n, m}:=H_{n} \times \mathbb{C}^{(m, n)} .
$$

We observe that $g=[\sigma, u,(\lambda, \mu, \kappa)]\left(\bmod \left\{ \pm E_{m+n}\right\}\right) \in N\left(H_{n}\right)$ acts on $H_{n, m}$ by

$$
(Z, W) \longmapsto\left(\sigma_{g}(Z), a(g ; Z)(W)+b(g ; Z)\right) .
$$

The subgroup of $N\left(H_{n}\right)$ consisting of elements $g=[\sigma, u,(\lambda, \mu, \kappa)]\left(\bmod \left\{ \pm E_{m+n}\right\}\right)$ with the property

$$
m_{g}=\text { Identity } \quad \text { on } H_{m}
$$


is called the Jacobi group, denoted by $G^{J}$. It follows immediately from the definition that

$$
G^{J}=\left\{\left[\sigma, E_{m},(\lambda, \mu, \kappa)\right] \in P\left(H_{n}\right)\right\} .
$$

It is easy to see that $G^{J}$ is the semidirect product of $S p(n, \mathbb{R})$ and $H_{\mathbb{R}}^{(n, m)}$, where

$$
H_{\mathbb{R}}^{(n, m)}:=\left\{\left[E_{n}, E_{m},(\lambda, \mu, \kappa)\right] \in P\left(H_{n}\right)\right\}
$$

is the nilpotent 2-step subgroup of $P\left(H_{n}\right)$, called the Heisenberg group. For some results on $H_{\mathbb{R}}^{(n, m)}$, we refer to [Y1]-[Y2].

Now we consider another subgroup $\tilde{G}$ of $G^{J}$. By the definition, $\tilde{G}$ consists of elements of $G^{J}$ whose action is of the following form:

$$
(Z, W, T) \longmapsto\left(\sigma_{g}(Z), a(g ; Z)(W), T+c(g ; Z, W)\right), \quad c(g ; Z, 0)=0 .
$$

LEMmA 2.2. The map

$$
J: \tilde{G} \times H_{n} \longrightarrow G L\left(\mathbb{C}^{(m, n)}\right)
$$

defined by

$$
J(\tilde{\sigma}, Z):=a(\tilde{\sigma} ; Z), \quad \tilde{\sigma} \in \tilde{G}, \quad Z \in H_{n}
$$

is a factor of automorphy for $\tilde{G}$.

Proof. It is easy to prove it. We leave its proof to the reader.

We note that the mapping

$$
A(g,(Z, W)):=c(g ; Z, W), \quad g \in G^{J},(Z, W) \in H_{n, m}
$$

is a summand of automorphy, i.e.,

$$
A\left(g_{1} g_{2},(Z, W)\right)=A\left(g_{1}, g_{2} \cdot(Z, W)\right)+A\left(g_{2},(Z, W)\right)
$$

where $g_{1}, g_{2} \in G^{J}$ and $(Z, W) \in H_{n, m}$. We let

$$
K_{\mathbb{C}} \subset G L\left(\mathbb{C}^{(m, n)}\right)
$$

be the complex Lie group generated by the linear mapping

$$
\left\{a(g ; Z): g \in G^{J}\right\} \text {. }
$$

Then $K_{\mathbb{C}}$ is isomorphic to $G L(n, \mathbb{C})$. 
LEMMA 2.3. Let

$$
\rho: G L(n, \mathbb{C}) \longrightarrow G L\left(V_{\rho}\right)
$$

be a finite dimensional holomorphic representation of $G L(n, \mathbb{C})$ on a finite dimensional complex vector space $V_{\rho}$ and let $\chi: \mathbb{C}^{(m, n)} \longrightarrow \mathbb{C}^{\times}$be a character on the additive group $\mathbb{C}^{(m, m)}$. Then the mapping $J_{\rho}: \tilde{G} \times H_{n} \longrightarrow G L\left(V_{\rho}\right)$ defined by

$$
J_{\rho}(\tilde{\sigma}, Z):=\rho(J(\tilde{\sigma}, Z)), \quad \tilde{\sigma} \in \tilde{G}, \quad Z \in H_{n}
$$

is a factor of automorphy for $\tilde{G}$. Furthermore the mapping

$$
J_{\chi, \rho}(g,(Z, W)):=\chi(c(g ; Z, W)) \rho(a(g ; Z)), \quad g \in G^{J}
$$

is a factor of automorphy for the Jacobi group $G^{J}$ with respect to $\chi$ and $\rho$.

Proof. The proof of this first statement is obvious. The proof of the second statement follows immediately from the fact that $A(g,(Z, W)):=c(g ; Z, W)$ is a summand of automorphy (cf. (2.1) and (2.2)) and that $J_{\rho}$ is a factor of automorphy for $\tilde{G}$.

DEFinition 2.4. $J_{\rho}$ and $J_{\chi, \rho}$ are called the canonical automorphic factor for $\tilde{G}$ with respect to $\rho$ and the canonical automorphic factor for $G^{J}$ with respect to $\chi$ and $\rho$ respectively.

\section{JACOBI Forms}

In this section, we establish the notations and define the concept of Jacobi forms.

Let

$$
S p(n, \mathbb{R})=\left\{M \in \mathbb{R}^{(2 n, 2 n)} \mid{ }^{t} M J_{n} M=J_{n}\right\}
$$

be the symplectic group of degree $n$, where

$$
J_{n}:=\left(\begin{array}{cc}
0 & E_{n} \\
-E_{n} & 0
\end{array}\right)
$$

It is easy to see that $S p(n, \mathbb{R})$ acts on $H_{n}$ transitively by

$$
M<Z>:=(A Z+B)(C Z+D)^{-1}
$$

where $M=\left(\begin{array}{cc}A & B \\ C & D\end{array}\right) \in S p(n, \mathbb{R})$ and $Z \in H_{n}$. 
For two positive integers $n$ and $m$, we recall that the Jacobi group $G^{J}:=$ $S p(n, \mathbb{R}) \ltimes H_{\mathbb{R}}^{(n, m)}$ is the semidirect product of the symplectic group $S p(n, \mathbb{R})$ and the Heisenberg group $H_{\mathbb{R}}^{(n, m)}$ endowed with the following multiplication law

$(M,(\lambda, \mu, \kappa)) \cdot\left(M^{\prime},\left(\lambda^{\prime}, \mu^{\prime}, \kappa^{\prime}\right)\right):=\left(M M^{\prime},\left(\tilde{\lambda}+\lambda^{\prime}, \tilde{\mu}+\mu^{\prime}, \kappa+\kappa^{\prime}+\tilde{\lambda}^{t} \mu^{\prime}-\tilde{\mu}^{t} \lambda^{\prime}\right)\right)$

with $M, M^{\prime} \in S p(n, \mathbb{R}),(\lambda, \mu, \kappa),\left(\lambda^{\prime}, \mu^{\prime}, \kappa^{\prime}\right) \in H_{\mathbb{R}}^{(n, m)}$ and $(\tilde{\lambda}, \tilde{\mu}):=(\lambda, \mu) M^{\prime}$. It is easy to see that $G^{J}$ acts on $H_{n, m}:=H_{n} \times \mathbb{C}^{(m, n)}$ transitively by

$$
(M,(\lambda, \mu, \kappa)) \cdot(Z, W):=\left(M<Z>,(W+\lambda Z+\mu)(C Z+D)^{-1}\right),
$$

where $M=\left(\begin{array}{ll}A & B \\ C & D\end{array}\right) \in S p(n, \mathbb{R}),(\lambda, \mu, \kappa) \in H_{\mathbb{R}}^{(n, m)}$ and $(Z, W) \in H_{n, m}$.

Let $\rho$ be a rational representation of $G L(n, \mathbb{C})$ on a finite dimensional complex vector space $V_{\rho}$. Let $\mathcal{M} \in \mathbb{R}^{(m, m)}$ be a symmetric half-integral semi-positive definite matrix of degree $m$. Let $C^{\infty}\left(H_{n, m}, V_{\rho}\right)$ be the algebra of all $C^{\infty}$ functions on $H_{n, m}$ with values in $V_{\rho}$. For $f \in C^{\infty}\left(H_{n, m}, V_{\rho}\right)$, we define

$$
\begin{aligned}
& \left(\left.f\right|_{\rho, \mathcal{M}}[(M,(\lambda, \mu, \kappa))]\right)(Z, W) \\
:= & e^{-2 \pi i \sigma\left(\mathcal{M}[W+\lambda Z+\mu](C Z+D)^{-1} C\right)} \times e^{2 \pi i \sigma\left(\mathcal{M}\left(\lambda Z^{t} \lambda+2 \lambda^{t} W+\left(\kappa+\mu^{t} \lambda\right)\right)\right)} \\
& \times \rho(C Z+D)^{-1} f\left(M<Z>,(W+\lambda Z+\mu)(C Z+D)^{-1}\right),
\end{aligned}
$$

where $M=\left(\begin{array}{cc}A & B \\ C & D\end{array}\right) \in S p(n, \mathbb{R}),(\lambda, \mu, \kappa) \in H_{\mathbb{R}}^{(n, m)}$ and $(Z, W) \in H_{n, m}$.

Definition 3.1. Let $\rho$ and $\mathcal{M}$ be as above. Let

$$
H_{\mathbb{Z}}^{(n, m)}:=\left\{(\lambda, \mu, \kappa) \in H_{\mathbb{R}}^{(n, m)} \mid \lambda, \mu \in \mathbb{Z}^{(m, n)}, \kappa \in \mathbb{Z}^{(m, m)}\right\} .
$$

A Jacobi form of index $\mathcal{M}$ with respect to $\rho$ on $\Gamma_{n}$ is a holomorphic function $f \in C^{\infty}\left(H_{n, m}, V_{\rho}\right)$ satisfying the following conditions (A) and (B):

(A) $\left.f\right|_{\rho, \mathcal{M}}[\tilde{\gamma}]=f$ for all $\tilde{\gamma} \in \Gamma_{n}^{J}:=\Gamma_{n} \ltimes H_{\mathbb{Z}}^{(n, m)}$.

(B) $f$ has a Fourier expansion of the following form :

$$
f(Z, W)=\sum_{\substack{T \geq 0 \\ \text { half-integral }}} \sum_{R \in \mathbb{Z}^{(n, m)}} c(T, R) \cdot e^{2 \pi i \sigma(T Z)} \cdot e^{2 \pi i \sigma(R W)}
$$

with $c(T, R) \neq 0$ only if $\left(\begin{array}{cc}T & \frac{1}{2} R \\ \frac{1}{2}^{t} R & \mathcal{M}\end{array}\right) \geq 0$.

If $n \geq 2$, the condition (B) is superfluous by Köcher principle ( cf. [Z] Lemma 1.6). We denote by $J_{\rho, \mathcal{M}}\left(\Gamma_{n}\right)$ the vector space of all Jacobi forms of index $\mathcal{M}$ with respect to $\rho$ on $\Gamma_{n}$. Ziegler ( cf. [Z] Theorem 1.8 or [E-Z] Theorem 1.1) proves that the vector space $J_{\rho, \mathcal{M}}\left(\Gamma_{n}\right)$ is finite dimensional. For more results on Jacobi forms with $n>1$ and $m>1$, we refer to [Y3]-[Y6] and [Z]. 


\section{Pluriharmonic Polynomials}

We review pluriharmonic polynomials of matrix arguments and collect some properties to be used in the next section (cf. $[\mathrm{K}-\mathrm{V}]$ and $[\mathrm{M}-\mathrm{N}-\mathrm{N}]$ ).

Let $n$ and $m$ be two positive integers and let $\mathcal{P}_{m, n}:=\mathbb{C}\left[W_{11}, W_{12}, \cdots, W_{m n}\right]$ be the ring of complex valued polynomials on $\mathbb{C}^{(m, n)}$. For any homogeneous polynomial $P \in \mathcal{P}_{m, n}$, we put

$$
P\left(\partial_{W}\right):=P\left(\frac{\partial}{\partial W_{11}}, \cdots, \frac{\partial}{\partial W_{m n}}\right) .
$$

Let $S$ be a positive definite symmetric rational matrix of degree $m$. Let $T:=\left(t_{p q}\right)$ be the inverse of $S$. For each $i, j$ with $1 \leq i, j \leq n$, we denote by $\triangle_{i, j}$ the following differential operator

$$
\triangle_{i, j}:=\sum_{p, q=1}^{m} t_{p q} \frac{\partial^{2}}{\partial W_{p i} \partial W_{q j}}, \quad 1 \leq i, j \leq n .
$$

A polynomial $P$ on $\mathbb{C}^{(m, n)}$ is said to be harmonic with respect to $S$ if

$$
\sum_{i=1}^{n} \triangle_{i, i} P=0
$$

A polynomial $P$ on $\mathbb{C}^{(m, n)}$ is called pluriharmonic with respect to $S$ if

$$
\triangle_{i, j} P=0, \quad 1 \leq i, j \leq n
$$

If there is no confusion, we just write harmonic or pluriharmonic instead of harmonic or pluriharmonic with respect to $S$. Obviously a pluriharmonic polynomial is harmonic. We denote by $\mathcal{H}_{m, n}$ the space of all pluriharmonic polynomials on $\mathbb{C}^{(m, n)}$. The ring $\mathcal{P}_{m, n}$ of polynomials on $\mathbb{C}^{(m, n)}$ has a symmetric nondegenerate bilinear form $\langle P, Q\rangle:=\left(P\left(\partial_{W}\right) Q\right)(0)$ for $P, Q \in \mathcal{P}_{m, n}$. It is easy to check that $<,>$ satisfies

$$
<P, Q R>=<Q\left(\partial_{W}\right) P, R>, \quad P, Q, R \in \mathcal{P}_{m, n}
$$

LEMmA 4.1. $\mathcal{H}_{m, n}$ is invariant under the action of $G L(n, \mathbb{C}) \times O(S)$ given by

$$
((A, B), P(W)) \longmapsto P\left({ }^{t} B W A\right), \quad A \in G L(n, \mathbb{C}), B \in O(S) .
$$

Here $O(S):=\left\{B \in G L(m, \mathbb{C}) \mid{ }^{t} B S B=S\right\}$ denotes the orthogonal group of the quadratic form $S$. 
Proof. See corollary 9.11 in [M-N-N].

Remark 4.2. In $[\mathrm{K}-\mathrm{V}]$, Kashiwara and Vergne investigated an irreducible decomposition of the space of complex pluriharmonic polynomials defined on $\mathbb{C}^{(m, n)}$ under the action of (4.6). They showed that each irreducible component $\tau \otimes \lambda$ occurring in the decomposition of $\mathcal{H}_{m, n}$ under the action (4.6) has multiplicity one and the irreducible representation $\tau$ of $G L(n, \mathbb{C})$ is determined uniquely by the irreducible representation of $O(S)$.

Lemma 4.3. If $P$ is pluriharmonic, then we have

$$
P\left(\partial_{W}\right) e^{\sigma\left(W C^{t} W S^{-1}\right)}=P\left(2 S^{-1} W C\right) e^{\sigma\left(W C^{t} W S^{-1}\right)}
$$

for all complex symmetric matrix $C \in \mathbb{C}^{(n, n)}$ of degree $n$. We recall that $\sigma(A)$ denotes the trace of a square matrix.

Proof. We set $h(W):=\sigma\left(W C^{t} W S^{-1}\right)$. We observe that $h\left(\partial_{W}\right) P=0$. Indeed,

$$
\begin{aligned}
h(W) & =\sum_{i, k, l, m} W_{i k} c_{k l} W_{m l} t_{m i} \\
& =\sum_{k, l} c_{k l}\left(\sum_{i, m} t_{m i} W_{m l} W_{i k}\right) \\
& =\sum_{k, l} c_{k l} h_{l k} .
\end{aligned}
$$

Thus $h\left(\partial_{W}\right) P=\sum_{k, l} c_{k l}\left(h_{l k}\left(\partial_{W}\right) P\right)=\sum_{k, l} c_{k l} \triangle_{l, k} P=0$. We put $\varphi(W):=$ $e^{h(W)}$. Then $f(W):=\varphi(W+A)=\varphi(W) \varphi(A) \eta(W)$, where $A \in \mathbb{C}^{(m, n)}$ and $\eta(W):=e^{\sigma\left(2 W C^{t} A S^{-1}\right)}$.

$$
\begin{aligned}
\left.P\left(\partial_{W}\right) \varphi(W)\right|_{W=A} & =\left.P\left(\partial_{W}\right) f(W)\right|_{W=0} \\
& =\left.\varphi(A)\left(P\left(\partial_{W}\right) \varphi(W) \eta(W)\right)\right|_{W=0} \\
& =\left.\varphi(A) P\left(\partial_{W}\right) \eta(W)\right|_{W=0} .
\end{aligned}
$$

Indeed, since $h\left(\partial_{W}\right) P=0$, we have

$$
\begin{aligned}
\left.P\left(\partial_{W}\right)(\varphi(W) \eta(W))\right|_{W=0} & =<P, \varphi \cdot \eta>=<\varphi\left(\partial_{W}\right) P, \eta> \\
& =\sum_{n=0}^{\infty} \frac{1}{n !}<h^{n}\left(\partial_{W}\right) P, \eta> \\
& =<P, \eta>=\left.P\left(\partial_{W}\right) \eta(W)\right|_{W=0} .
\end{aligned}
$$


By an easy computation, we obtain

$$
P\left(\partial_{W}\right) \eta(W)=P\left(2 S^{-1} A C\right) \eta(W) .
$$

Finally, we have

$$
\left.P\left(\partial_{W}\right) \varphi(W)\right|_{W=A}=\varphi(A) \cdot P\left(2 S^{-1} A C\right) \eta(0)
$$

Hence we obtain the desired result.

\section{Proof of Main Theorem}

Throughout this section we fix a rational representation $\rho$ of $G L(n, \mathbb{C})$ on a finite dimensional complex vector space $V_{\rho}$ and a positive definite symmetric, halfintegral matrix $\mathcal{M}$ of degree $m$ once and for all.

We set $S:=(2 \mathcal{M})^{-1}$. As in the previous section, we denote by $\mathcal{H}_{m, n}$ the vector space of all pluriharmonic polynomials with respect to $S$ on $\mathbb{C}^{(m, n)}$. According to Lemma 4.1, there exists an irreducible subspace $V_{\tau}(\neq 0)$ invariant under the action of $G L(n, \mathbb{C})$ given by $(4.6)$. We denote this represetation by $\tau$. Then we have

$$
(\tau(A) P)(W)=P(W A), \quad A \in G L(n, \mathbb{C}), \quad P \in V_{\tau}, \quad W \in \mathbb{C}^{(m, n)} .
$$

The action $\hat{\tau}$ of $G L(n, \mathbb{C})$ on $V_{\tau}^{*}$ is defined by

$$
\left(\hat{\tau}(A)^{-1} \zeta\right)(P):=\zeta\left(\tau\left({ }^{t} A^{-1}\right) P\right)
$$

where $A \in G L(n, \mathbb{C}), \zeta \in V_{\tau}^{*}$ and $P \in V_{\tau}$.

Definition 5.1. Let $f \in J_{\rho, \mathcal{M}}\left(\Gamma_{n}\right)$ be a Jacobi form of index $\mathcal{M}$ with respect to $\rho$ on $\Gamma_{n}$. Let $P \in V_{\tau}$ be a homogeneous pluriharmonic polynomial. We put

$$
f_{P}(Z):=\left.P\left(\partial_{W}\right) f(Z, W)\right|_{W=0}, \quad Z \in H_{n}, \quad W \in \mathbb{C}^{(m, n)} .
$$

Now we define the mapping

$$
f_{\tau}: H_{n} \longrightarrow V_{\tau}^{*} \otimes V_{\rho}
$$

by

$$
\left(f_{\tau}(Z)\right)(P):=f_{P}(Z), \quad Z \in H_{n}, \quad P \in V_{\tau} .
$$


Definition 5.2. A holomorphic function $f: H_{n} \rightarrow V_{\rho}$ is called a modular form of type $\rho$ on $\Gamma_{n}$ if

$$
f(M<Z>)=\rho(C Z+D) f(Z), \quad Z \in H_{n}
$$

for all $M=\left(\begin{array}{cc}A & B \\ C & D\end{array}\right) \in \Gamma_{n}$. If $n=1$, the additional cuspidal condition will be added. We denote by $\left[\Gamma_{n}, \rho\right]$ the vector space of all modular forms of type $\rho$ on $\Gamma_{n}$.

Main Theorem. Let $\tau$ and $\hat{\tau}$ be as before. Let $f \in J_{\rho, \mathcal{M}}\left(\Gamma_{n}\right)$ be a Jacobi form. Then $f_{\tau}(Z)$ is a modular form of type $\hat{\tau} \otimes \rho$, i.e., $f_{\tau} \in\left[\Gamma_{n}, \hat{\tau} \otimes \rho\right]$.

Proof. Let

$$
f(Z, W)=\sum_{T, R} c(T, R) e^{2 \pi i \sigma(T Z)} \cdot e^{2 \pi i \sigma(R W)}
$$

be a Fourier expansion of $f(Z, W)$. Then we have

$$
P\left(\partial_{W}\right) f(Z, W)=\sum_{T, R} P\left(2 \pi i^{t} R\right) \cdot c(T, R) \cdot e^{2 \pi i \sigma(T Z+R W)}
$$

and

$$
f_{P}(Z):=\left.P\left(\partial_{W}\right) f(Z, W)\right|_{W=0}=\sum_{T, R} P\left(2 \pi i^{t} R\right) \cdot e^{2 \pi i \sigma(T Z)} \cdot c(T, R)
$$

Since $f \in J_{\rho, \mathcal{M}}\left(\Gamma_{n}\right)$, we have the following transformation law

$$
f\left(M<Z>, W(C Z+D)^{-1}\right)=e^{2 \pi i \sigma\left(\mathcal{M} W(C Z+D)^{-1} C^{t} W\right)} \cdot \rho(C Z+D) f(Z, W)
$$

for all $M=\left(\begin{array}{cc}A & B \\ C & D\end{array}\right) \in \Gamma_{n}$. Applying $P\left(\partial_{W}\right)$ to $(5.6)$, according to Lemma 4.3, we have

$$
\begin{aligned}
& P\left(\partial_{W}\right) f\left(M<Z>, W(C Z+D)^{-1}\right) \\
= & P\left(4 \pi i \mathcal{M} W(C Z+D)^{-1} C\right) e^{2 \pi i \sigma\left(\mathcal{M} W(C Z+D)^{-1} C^{t} W\right)} \\
& \times \rho(C Z+D) f(Z, W)+h(Z, W)+e^{2 \pi i \sigma\left(\mathcal{M} W(C Z+D)^{-1} C^{t} W\right)} \\
& \times \sum_{T, R} P\left(2 \pi i^{t} R\right) \cdot \rho(C Z+D) c(T, R) \cdot e^{2 \pi i \sigma(T Z+R W)},
\end{aligned}
$$

where $h(Z, W)$ is a $V_{\rho}$-valued function on $H_{n, m}$ whose restriction to $W=0$ vanishes. Here we used the fact that $(C Z+D)^{-1} C$ is a complex symmetric matrix 
of degree $n$ and Lemma 4.3. If we evaluate this at $W=0, P$ being homogeneous, we have

$$
\begin{aligned}
& \left.P\left(\partial_{W}\right) f\left(M<Z>, W(C Z+D)^{-1}\right)\right|_{W=0} \\
= & \sum_{T, R} P\left(2 \pi i^{t} R\right) \cdot e^{2 \pi i \sigma(T Z)} \cdot \rho(C Z+D) c(T, R) .
\end{aligned}
$$

On the other hand,

$$
\begin{aligned}
& \left.P\left(\partial_{W}\right) f\left(M<Z>, W(C Z+D)^{-1}\right)\right|_{W=0} \\
= & \left.P\left(\partial_{W}\right) \sum_{T, R} c(T, R) e^{2 \pi i \sigma(T \cdot M<Z>)} \cdot e^{2 \pi i \sigma\left(R W(C Z+D)^{-1}\right)}\right|_{W=0} \\
= & \sum_{T, R} P\left(2 \pi i^{t} R^{t}(C Z+D)^{-1}\right) \cdot e^{2 \pi i \sigma(T \cdot M<Z>)} \cdot c(T, R) .
\end{aligned}
$$

Thus according to (5.7), we have

$$
\begin{aligned}
& \sum_{T, R} \tilde{P}\left(2 \pi i^{t} R\right) \cdot e^{2 \pi i \sigma(T \cdot M<Z>)} \cdot c(T, R) \\
= & \sum_{T, R} P\left(2 \pi i^{t} R\right) \cdot e^{2 \pi i \sigma(T Z)} \cdot \rho(C Z+D) c(T, R),
\end{aligned}
$$

where $\tilde{P}(W):=P\left(W^{t}(C Z+D)^{-1}\right)$. By (5.5), (5.8) implies

$$
f_{\tilde{P}}(M<Z>)=\rho(C Z+D) f_{P}(Z),
$$

that is,

$$
\left(f_{\tau}(M<Z>)\right)(\tilde{P})=\rho(C Z+D) f_{\tau}(Z)(P) .
$$

Since $\tilde{P}=\tau\left({ }^{t}(C Z+D)^{-1}\right) P$, we have from $(5.9)$

$$
\begin{aligned}
& \left(\left(\hat{\tau}^{-1} \otimes 1_{V_{\rho}}\right)(C Z+D) f_{\tau}(M<Z>)\right)(P) \\
= & \left(\left(1_{V_{\tau} *} \otimes \rho\right)(C Z+D) f_{\tau}(Z)\right)(P),
\end{aligned}
$$

where $1_{V_{\tau}}\left(\right.$ resp. $\left.V_{\rho}\right)$ denotes the trivial representation of $G L(n, \mathbb{C})$ on $V_{\tau}^{*}\left(\right.$ resp. $\left.V_{\rho}\right)$. Hence we obtain

$$
f_{\tau}(M<Z>)=(\hat{\tau} \otimes \rho)(C Z+D) f_{\tau}(Z)
$$

for all $M=\left(\begin{array}{cc}A & B \\ C & D\end{array}\right) \in \Gamma_{n}$. Therefore $f_{\tau}$ is a $\operatorname{Hom}\left(V_{\tau}, V_{\rho}\right)$-valued modular form of type $\hat{\tau} \otimes \rho$. 


\section{Applications}

In this final section, we obtain important identites by applying the main theorem to two special Jacobi forms.

(I) Let $S \in \mathbb{Z}^{(2 k, 2 k)}$ be a positive definite symmetirc, unimodular even matrix of degree $2 k$. We choose an integral matrix $c \in \mathbb{Z}^{(2 k, m)}$ such that ${ }^{t} c S c$ is positive definite. We consider the following theta series

$$
\theta_{S, c}(Z, W):=\sum_{\lambda \in \mathbb{Z}^{(2 k, n)}} e^{\pi i \sigma\left(S\left(\lambda Z^{t} \lambda+2 \lambda^{t}(c W)\right)\right)} .
$$

Then $\theta_{S, c} \in J_{k, \mathcal{M}}\left(\Gamma_{n}\right)$ with $\mathcal{M}:=\frac{1}{2}{ }^{t} c S c$ (cf. [Z], p. 212). We write $f(Z, W):=$ $\theta_{S, c}(Z, W)$. Then by Main Theorem, $f_{\tau}$ is a $\operatorname{Hom}\left(V_{\tau}, \mathbb{C}\right)$-valued modular form of type $\hat{\tau} \otimes \operatorname{det}^{k}$. Furthermore, according to (5.9), for any homogeneous pluriharmonic $P$ with respect to $(2 \mathcal{M})^{-1}=\left({ }^{t} C S c\right)^{-1}$, we obtain the following identity

$$
\begin{aligned}
& \sum_{\lambda \in \mathbb{Z}^{(2 k, n)}} P\left(2 \pi i^{t} c S \lambda^{t}(C Z+D)^{-1}\right) \cdot e^{\pi i \sigma\left(S \lambda(A Z+B)(C Z+D)^{-1} t \lambda\right)} \\
= & \{\operatorname{det}(C Z+D)\}^{k} \sum_{\lambda \in \mathbb{Z}^{(2 k, n)}} P\left(2 \pi i^{t} c S \lambda\right) \cdot e^{\pi i \sigma\left(S \lambda Z^{t} \lambda\right)}
\end{aligned}
$$

for all $M=\left(\begin{array}{cc}A & B \\ C & D\end{array}\right) \in \Gamma_{n}$ and $Z \in H_{n}$.

(II) In [Z], Ziegler defined the Eisenstein series $E_{k, \mathcal{M}}^{(n)}(Z, W)$ of Siegel type. Let $\mathcal{M}$ be a half integral positive definite symmetric matrix of degree $m$ and let $k \in \mathbb{Z}^{+}$. We set

$$
\Gamma_{n, 0}:=\left\{\left(\begin{array}{cc}
A & B \\
C & D
\end{array}\right) \in \Gamma_{n} \mid C=0\right\} .
$$

Let $\mathcal{R}$ be a complete system of representatives of the cosets $\Gamma_{n, 0} \backslash \Gamma_{n}$ and $\Lambda$ be a complete system of representatives of the cosets $\mathbb{Z}^{(m, n)} /\left(\operatorname{ker}(\mathcal{M}) \cap \mathbb{Z}^{(m, n)}\right)$, where $\operatorname{ker}(\mathcal{M}):=\left\{\lambda \in \mathbb{R}^{(m, n)} \mid \mathcal{M} \cdot \lambda=0\right\}$. The Eisenstein series $E_{k, \mathcal{M}}^{(n)}$ is defined by

$$
\begin{aligned}
E_{k, \mathcal{M}}^{(n)}(Z, W):= & \sum \operatorname{det}(C Z+D)^{-k} \cdot e^{2 \pi i \sigma\left(\mathcal{M} W(C Z+D)^{-1} C^{t} W\right)} \\
& \left.\cdot \begin{array}{ll}
C & D
\end{array}\right) \in \mathcal{R} \\
& \cdot \sum_{\lambda \in \Lambda} e^{2 \pi i \sigma\left(\mathcal{M}\left((A Z+B)(C Z+D)^{-1 t} \lambda+2 \lambda^{t}(C Z+D)^{-1 t} W\right)\right)}
\end{aligned}
$$

where $(Z, W) \in H_{n, m}$. Now we assume that $k>n+m+1$ and $k$ is even. Then according to $[Z]$, Theorem $2.1, E_{k, \mathcal{M}}^{(n)}(Z, W)$ is a nonvanishing Jacobi form in 
$J_{k, \mathcal{M}}\left(\Gamma_{n}\right)$. By Main Theorem, $\left(E_{k, \mathcal{M}}^{(n)}\right)_{\tau}$ is a $\operatorname{Hom}\left(V_{\tau}, \mathbb{C}\right)$-valued modular form of type $\hat{\tau} \otimes \operatorname{det}^{k}$. We define the automorphic factor $j: S p(n, \mathbb{R}) \times H_{n} \longrightarrow G L(n, \mathbb{C})$ by

$$
j(g, Z):=c Z+d, \quad g=\left(\begin{array}{ll}
a & b \\
c & d
\end{array}\right) \in S p(n, \mathbb{R}), \quad Z \in H_{n} .
$$

Then according to (5.9), for any homogeneous pluriharmonic polynomial $P$ with respect to $(2 \mathcal{M})^{-1}$, we obtain the following identity

$$
\begin{aligned}
& \operatorname{det} j(M, Z)^{k} \sum_{\gamma \in \mathcal{R}} \sum_{\lambda \in \Lambda} \operatorname{det} j(\gamma, Z)^{-k} \cdot P\left(4 \pi i \mathcal{M} \lambda^{t} j(\gamma, Z)^{-1}\right) \cdot e^{2 \pi i \sigma\left(\mathcal{M} \cdot \gamma<Z>{ }^{t} \lambda\right)} \\
= & \sum_{\gamma \in \mathcal{R}} \sum_{\lambda \in \Lambda} \operatorname{det} j(\gamma, M<Z>)^{-k} \cdot P\left(4 \pi i \mathcal{M} \lambda^{t} j(\gamma M, Z)^{-1}\right) \cdot e^{2 \pi i \sigma\left(\mathcal{M} \cdot \gamma M<Z>{ }^{t} \lambda\right)}
\end{aligned}
$$

for all $M \in \Gamma_{n}$ and $Z \in H_{n}$.

ACKnowledgements: This work was done in part during my stay at the MaxPlanck-Institut für Mathematik. I am very grateful to the institute for the hospitality and support.

\section{REFERENCES}

[E-Z] M. Eichler and D. Zagier, The Theory of Jacobi Forms, Progress in Math., vol. 55, Birkhäuser, Boston-Basel- Stuttgart, 1985.

[K-V] M. Kashiwara and M. Vergne, On the Segal-Shale-Weil Representations and Harmonic Polynomials, Invent. Math. 44 (1978), 1-47.

[M-N-N] D. Mumford, M. Nori and P. Norman, Tata Lectures on Theta III, Progress in Math., vol. 97, Birkhäuser, 1991.

[N] Y. Namikawa, Toroidal compactification of Siegel spaces, vol. 812, Springer-Verlag, 1980.

[PS] I. Piateski-Sharpiro, Automorphic Functions and the Geometry of Classical Domains, Gordan-Breach, New York, 1966.

[Y1] J.-H. Yang, Harmonic Analysis on the Quotient Spaces of Heisenberg Groups, Nagoya Math. J. 123 (1991), 103-117.

[Y2] Harmonic Analysis on the Quotient Spaces of Heisenberg Groups II, J. Number Theory 49, No. 1 (1994), 63-72.

[Y3] _ Some Results on Jacobi Forms of Higher Degree, Proc. RIMS Symp. on Automorphic Forms and Associated Zeta Functions, Kyoto 805 (1992), 36-50.

[Y4] The Siegel-Jacobi Operator, Abh. Math. Sem. Univ. Hamburg 63 (1993), $135-146$.

[Y5] L Remarks on Jacobi Forms of Higher Degree, Proc. of the 1993 Workshop on Automorphic Forms and Related Topics, The Pyungsan Institute for Mathematical Sciences, Seoul (1993), 33-58.

[Y6] Singular Jacobi Forms, to appear in Trans. of American Math. Soc. 
[Z] C. Ziegler, Jacobi Forms of Higher Degree, Abh. Math. Sem. Univ. Hamburg 59 (1989), 191-224.

Department of Mathematics

INHA UNIVERSITY

INCHEON 402-751

REPUBLIC OF KOREA

email address: jhyang@inha.ac.kr 